\title{
Donor human milk may decrease severe gastrointestinal distress in infants with neonatal abstinence syndrome.
}

\author{
Catherine Alexander, Paula Radmacher*, Lori Devlin \\ Department of Pediatrics, Division of Neonatal Medicine, University of Louisville School of Medicine, USA
}

\begin{abstract}
Introduction: Neonatal Abstinence Syndrome (NAS) is a drug withdrawal syndrome that occurs after antenatal exposure to opiates. Symptoms generally peak at 48-72 after delivery. One of the postnatal challenges for infants with NAS is feeding intolerance. The use of mother's own milk has been reported to improve NAS symptoms when compared to formula. However, the American Academy of Pediatrics (AAP) considers the unsupervised use of opiates and other street drugs to be a contraindication to breastfeeding. We wanted to test the efficacy of donor human milk to ease gastrointestinal symptoms in infants with NAS who cannot receive their mother's milk.

Methods: Infants whose initial withdrawal symptoms had been stabilized on morphine and whose mothers were not providing their milk were eligible to be enrolled. Infants were fed only donor human milk (DHM) for a period of up to 2 weeks. Finnegan scores, oral morphine dosing, and adjuvant therapy were prescribed according to a standardized protocol. Twelve infants were enrolled in the study and 9 infants completed at least 7 days of exclusive DHM feedings. Infants were matched 1:1 to historic controls with similar perinatal exposure who were formula-fed.

Results: Median Finnegan scores were not statistically different between the study and control groups. However, the proportion of infants with GI sub-scores greater than 2 was significantly higher in the control group when compared to infants who received DHM $(p=0.001)$.

Conclusions: The present pilot study suggests that feeding with DHM may decrease severe GI distress for infants with NAS.
\end{abstract}

Keywords: Donor human milk, Neonatal abstinence syndrome, Infants.

\section{Introduction}

Neonatal Abstinence Syndrome (NAS) is a drug withdrawal syndrome that occurs primarily after antenatal exposure to opiates. The incidence of NAS and the need for pharmacological treatment has increased substantially over the last decade [1]. Withdrawal symptoms may present at birth, but often peak at 48-72 hours of life.

Withdrawal is elicited by the precipitous removal of exogenous opiates at the time of delivery which leads to significant increases in norepinephrine levels resulting in marked sympathetic upregulation [2]. The onset of symptoms is affected by the half-life of the opiate(s) used during pregnancy, exposure to other mind altering prescription and/or illicit drugs, differences in maternal and infant metabolism, and genetic and epigenetic factors [3-6]. Neonatal withdrawal symptoms are directly linked to the location of mu opioid receptors, which are concentrated in the brain but are also found in the sensory nerves, mast cells, and in the GI tract [2]. The Finnegan Neonatal Abstinence Scoring System has been used for the last 40 years [7-11]. to assess the severity of neonatal withdrawal after in-utero opioid exposure. Within the total Finnegan assessment, 5 different gastrointestinal symptoms are scored from 0-3 depending on severity.
One of the challenges for infants with NAS is feeding intolerance [1]. Human milk has been shown to be better tolerated than formula in most infants and is cited by the AAP as the preferred feeding method for all infants [12] Human milk contains hormones, immunoglobulins, and growth factors that stimulate gastrointestinal growth and motility. It has been shown to increase gastric emptying and lactase activity in the gut which also improve feeding tolerance [13-15]. The intake of mother's own milk has been found to improve attachment, reduce severity of withdrawal symptoms, decrease need for and shorten the length of pharmacological treatment for infants with NAS [7,16-19]. However, the unsupervised use of opiates alone or in conjunction with other mind altering prescription or street drugs is a proposed contraindication to the use of mother's own milk [18]. No reports to date have evaluated the effect of donor human milk (DHM) on infants undergoing opiate withdrawal who cannot or do not receive their mother's own milk.

The aim of this study was to test the feasibility of using DHM as a non-pharmacologic adjunct therapy to ease GI symptoms of infants undergoing pharmacologic treatment for NAS who could not receive their own mother's milk. 


\section{Methods}

This study was approved by the University of Louisville IRB and was conducted in the NICU at Norton Children's and the University of Louisville Hospitals. Women with perinatal drug exposure were approached about their infant's participation only if breastfeeding was deemed by the attending neonatologist to be contraindicated due to illicit drug use in the six weeks prior to delivery or if the mother had clearly established that she preferred formula as the feeding choice for her infant. Maternal informed consent was obtained prior to any study procedures. Infants were eligible for the study if they were term $(>370 / 7$ weeks gestation), appropriate for gestational age (AGA), known to have perinatal opiate exposure and receiving oral morphine according to a local protocol to manage the initiation, escalation and weaning of pharmacologic therapy (Figure 1). Study enrollment occurred once infants were captured on oral morphine. Infants were considered captured if their total Finnegan scores (Table 1) were improving and they were deemed ready for weaning by the clinical team.

After consent was obtained, infants were exclusively fed DHM (Co-op Donor Milk, Medolac Laboratories, Lake Oswego, OR) for a period of up to 2 weeks. All total Finnegan scores as well as GI sub-scores were recorded. A GI sub-score greater than 2 was considered to represent significant GI disturbance. Maternal demographic data included: age, gravidity and parity, drug history, and toxicology results. Infant demographic information included: birth weight, head circumference, sex, ethnicity, mode of delivery, daily weights, morphine dosing, need for and dosing of adjuvant therapy, stool characteristics, length of stay and length of treatment. Study infants were matched one to one with recent historic controls who had similar drug exposure profiles and were primarily formula fed.

Data between the study infants and controls were compared by using a student t-test and $\mathrm{X}^{2}$ using SPSS V.24. Statistical significance was set at $\mathrm{p}<0.05$.

\section{Results}

Twelve infants were enrolled in the study and 9 infants completed at least 7 days of exclusive donor milk feedings. In-utero drug exposure was similar between groups (Table 2). Benzodiazepine exposure was noted in the donor human milk group but not in the control group.

Birth anthropometrics and maternal age were similar between groups (Table 3). Of note, the $\mathrm{HC}$ in the historical controls was statistically smaller (but still appropriate for gestational age) than the study infants $(p=0.023)$. Infants in both groups were captured on morphine therapy at a mean of 5.6 days of life (Table 4). There was an $11 \%$ reduction in the need for adjuvant therapy (56.5\% vs. $44.4 \%$, control vs. treatment) which did not meet statistical significance. Return to birth weight and daily weight gain during the study period were similar between groups. The head circumference gain in the study infants was slightly less than that of the control group $(\mathrm{p}=0.025)$.

Median total Finnegan scores were not statistically different between the groups. However, there was a trend toward lower GI sub-scores in the DHM group as shown in Figure 2. The proportion of infants with GI sub-scores greater than 2, suggesting more intense GI distress, was significantly higher in the control group when compared to infants who received DHM

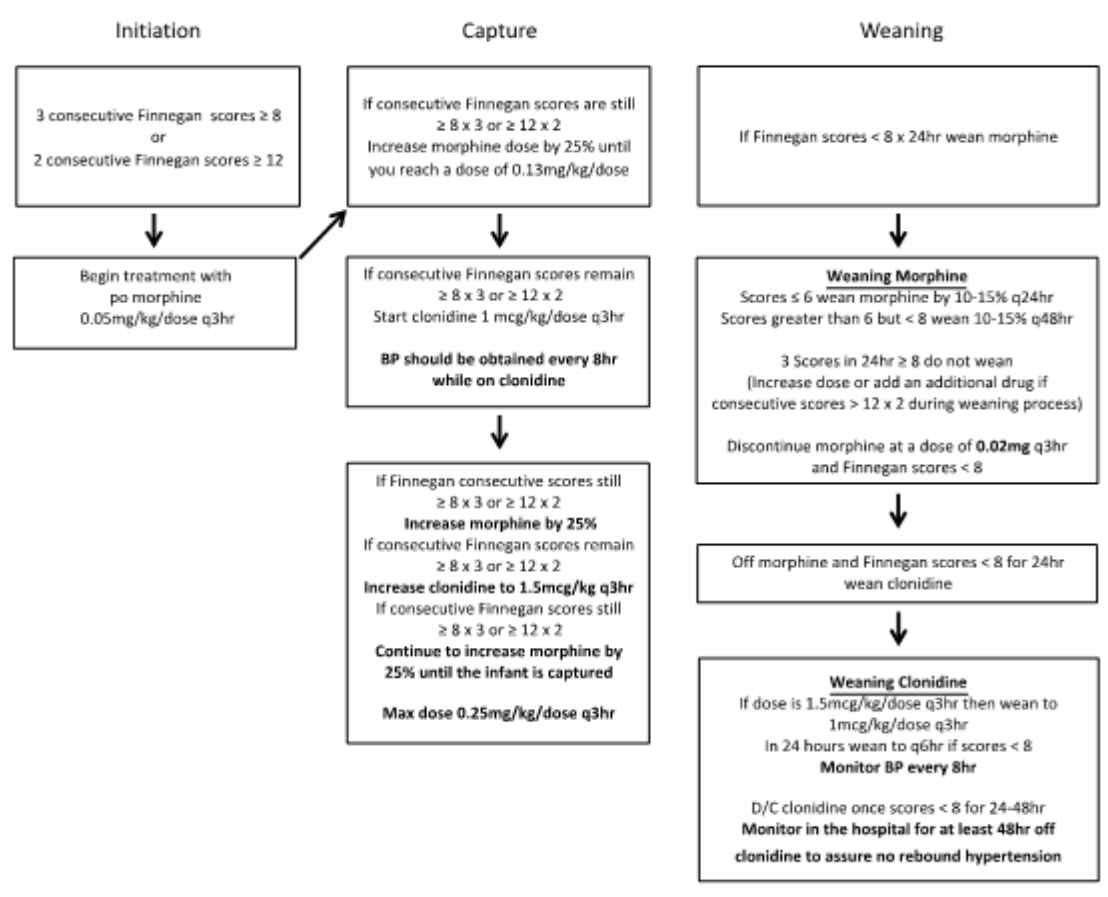

Abbreviations: mg/kg, milligrams per kilogram; q, every; hr, hours; po, by mouth; BP, blood pressure

Figure 1. Treatment protocol for Neonatal Abstinence Syndrome (NAS). 
Table 1. Finnegan scoring algorithm [11].

\begin{tabular}{|c|c|c|}
\hline System & Signs \& Symptoms & Score \\
\hline \multirow{8}{*}{ 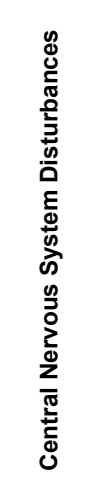 } & $\begin{array}{l}\text { Excessive high-pitched (or other) cry }<5 \mathrm{~min} \\
\text { Continuous high-pitched (or other) cry }>5 \mathrm{~min}\end{array}$ & $\begin{array}{l}2 \\
3\end{array}$ \\
\hline & $\begin{array}{l}\text { Sleeps }<1 \text { hour after feeding } \\
\text { Sleeps }<2 \text { hours after feeding } \\
\text { Sleeps }<3 \text { hours after feeding }\end{array}$ & $\begin{array}{l}3 \\
2 \\
1\end{array}$ \\
\hline & $\begin{array}{l}\text { Hyperactive Moro reflex } \\
\text { Markedly hyperactive Moro reflex }\end{array}$ & $\begin{array}{l}2 \\
3\end{array}$ \\
\hline & $\begin{array}{l}\text { Mild tremors when disturbed } \\
\text { Moderate-severe tremors when disturbed } \\
\text { Mild tremors when undisturbed } \\
\text { Moderate-severe tremors when undisturbed }\end{array}$ & $\begin{array}{l}1 \\
2 \\
3 \\
4\end{array}$ \\
\hline & Increased muscle tone & 1 \\
\hline & Excoriation (chin, knees, elbow, toes, nose) & 1 \\
\hline & Myoclonic jerks (twitching/jerking of limbs) & 3 \\
\hline & Generalized convulsions & 5 \\
\hline \multirow{10}{*}{ 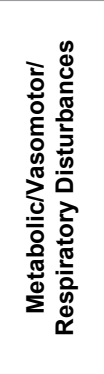 } & Sweating & 1 \\
\hline & Hyperthermia $37.2-38.3^{\circ} \mathrm{C}$ & 1 \\
\hline & Hyperthermia $>38.4^{\circ} \mathrm{C}$ & 2 \\
\hline & Frequent yawning (>3-4 times/scoring interval) & 1 \\
\hline & Mottling & 1 \\
\hline & Nasal stuffiness & 1 \\
\hline & Sneezing (>3-4 times/scoring interval) & 1 \\
\hline & Nasal flaring & 2 \\
\hline & Respiratory rate $>60 / \mathrm{min}$ & 1 \\
\hline & Respiratory rate $>60 / \mathrm{min}$ with retractions & 2 \\
\hline \multirow{5}{*}{ 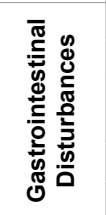 } & Excessive sucking & 1 \\
\hline & Poor feeding (infrequent/uncoordinated suck) & 2 \\
\hline & $\begin{array}{c}\text { Regurgitation ( } \geq 2 \text { times during/post feeding) } \\
\text { Projectile vomiting }\end{array}$ & $\begin{array}{l}2 \\
3\end{array}$ \\
\hline & Loose stools (curds/seeding appearance) & 2 \\
\hline & Watery stools (water ring on diaper around stool) & 3 \\
\hline $\begin{array}{l}\text { Total } \\
\text { score }\end{array}$ & Maximum score & - \\
\hline
\end{tabular}

Table 2. Maternal drugs.

\begin{tabular}{|c|c|c|}
\hline & Donor milk & Control Group \\
\hline Heroin & 4 & 7 \\
\hline Buprenorphine & 4 & 4 \\
\hline Methadone & 1 & 3 \\
\hline Other opiates & 1 & 1 \\
\hline Marijuana & 2 & 1 \\
\hline Benzodiazepines & 2 & 0 \\
\hline Ethanol & 1 & 0 \\
\hline
\end{tabular}

Table 3. Demographic data.

\begin{tabular}{|c|c|c|c|}
\hline & $\begin{array}{c}\text { Donor milk } \\
(\mathbf{n = 9})\end{array}$ & $\begin{array}{c}\text { Control } \\
(\mathbf{n = 9})\end{array}$ & $\mathbf{p}$ \\
\hline Birth weight (grams) & $3091 \pm 350$ & $2934 \pm 495$ & NS \\
\hline Gestational age (wks) & $38.8 \pm 1.0$ & $38.8 \pm 1.4$ & NS \\
\hline Head circumference @ birth (cm) & $34.1 \pm 1.2$ & $32.6 \pm 1.3$ & $0.023^{*}$ \\
\hline Male & $77.8 \%$ & $44.4 \%$ & NS \\
\hline Maternal age (yrs) & $27 \pm 5$ & $23 \pm 4$ & NS \\
\hline
\end{tabular}

* AGA 38 weeks gestation-Olsen et al. Pediatrics 2010; 125:e214-224

Table 4. Infant NAS course.

\begin{tabular}{|c|c|c|c|}
\hline & $\begin{array}{c}\text { Donor milk } \\
(\mathbf{n = 9})\end{array}$ & $\begin{array}{c}\text { Control } \\
(\mathbf{n = 9})\end{array}$ & $\mathbf{p}$ \\
\hline Age @ capture (days) & $5.6 \pm 1.7$ & $5.6 \pm 2.1$ & NS \\
\hline Return to BW (days) & $17 \pm 7$ & $16 \pm 4$ & NS \\
\hline Length of treatment (days) & $37 \pm 16$ & $29 \pm 9$ & NS \\
\hline Required more than 1 medication to treat & $44.4 \%$ & $55.6 \%$ & NS \\
\hline Length of stay (days) & $41 \pm 16$ & $33 \pm 9$ & NS \\
\hline Weight gain during study period (g/day) & $19.1 \pm 13.1$ & $20.5 \pm 3.0$ & NS \\
\hline Head circumference gain (cm/wk) & $0.4 \pm 0.1$ & $0.6 \pm 0.2$ & 0.025 \\
\hline GI Sub-scores $>2$ & $39 \%$ & $61 \%$ & 0.001 \\
\hline
\end{tabular}

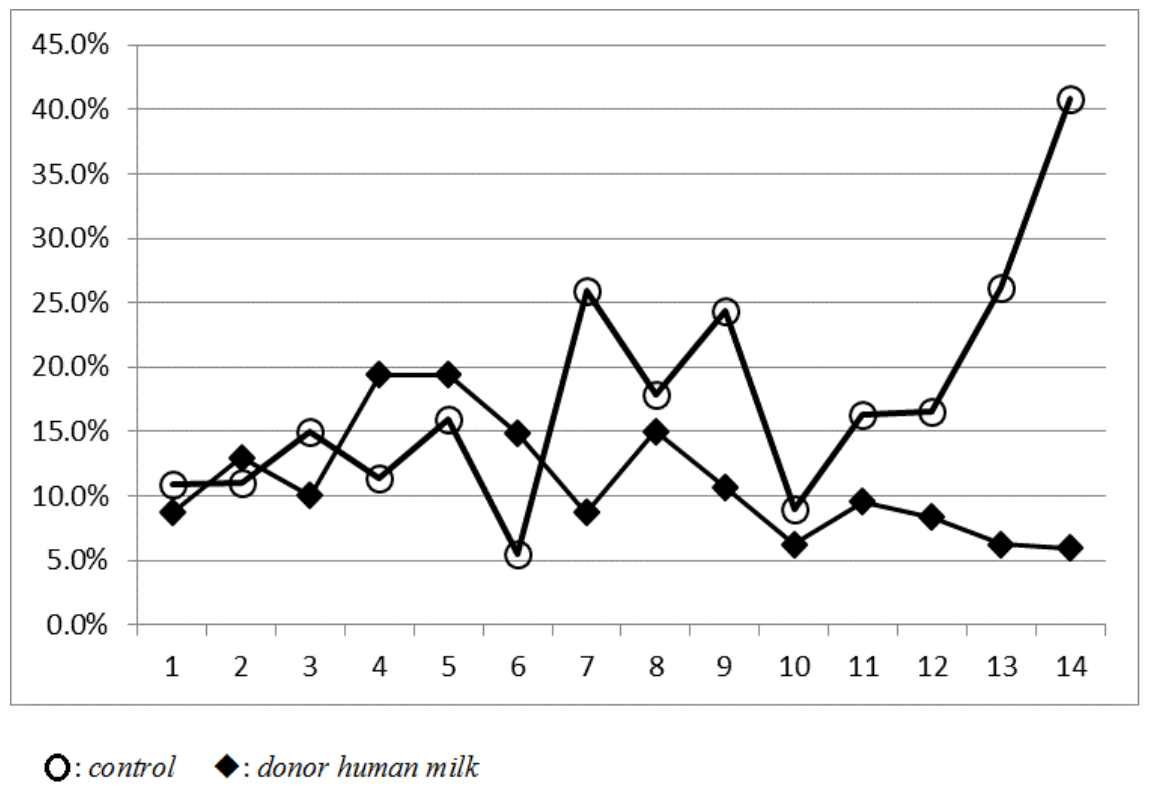

Figure 2. Mean proportion of GI sub-scores (>2) by group and study day.

(Table $4, p=0.001$ ). No statistical difference was noted in length of treatment or length of stay.

\section{Discussion}

In this feasibility study of term infants with NAS, those fed an exclusive diet of DHM during the study period had signiicantly less severe GI distress than control infants fed primarily with term formula. This inding suggests that DHM has some capacity to calm the GI tract during the acute withdrawal period after perinatal opioid exposure.

Previous studies evaluating outcomes for infants who received their mother's own milk have noted similar findings, although the amount of opiates passed through breast milk is relatively 
small [18]. Jansson found the average amount of methadone transferred through mother's own milk $<0.2 \mathrm{mg}$ /day at day of life 30 [20]. Liquid chromatography-tandem mass spectrometry found low norbuprenorphine concentrations $(<3.7 \mathrm{ug} / \mathrm{L})$ in mother's own milk, which correlated with buprenorphine levels in infant plasma of $<0.7 \mathrm{ug} / \mathrm{L} \rightarrow[21,22]$. Complete delineation of the impact of human milk from the effects of passively transferred opiates in the milk was not possible in these studies. The findings of our study suggest that the innate properties of human milk may independently modulate the severity of GI distress in infants with NAS.

Weight gain was similar between infants fed DHM and formula. However, HC growth appeared to be slower in the DHM group. This finding requires further study. A trend toward a decrease in the need for adjuvant therapy was noted in the DHM group although this did not meet statistical significance.

Length of treatment and length of stay were not impacted by the administration of an exclusive DHM diet during the first 1-2 weeks of treatment for NAS. This was not unexpected as infants did not receive DHM throughout their entire hospital stay. This study was designed to test the efficacy of DHM as a non-pharmacologic adjunct treatment and as such it was not powered for such outcomes.

The study was limited by the small number of infants. Enrollment was complicated by a number of factors, including complex maternal social issues and a general unfamiliarity with using donor human milk as a feeding option.

\section{Conclusion}

The findings of this study suggest that DHM may improve severe GI symptoms. DHM may enhance non-pharmacologic intervention for infants with NAS and play a role in moderating acute withdrawal after perinatal opioid exposure. A larger clinical trial using DHM throughout the treatment period is needed to confirm the findings of this pilot study.

\section{Acknowledgement}

Medolac Laboratories provided the milk for this study.

\section{References}

1. Patrick SW, Schumacher RE, Benneyworth BD, et al. Neonatal abstinence syndrome and associated health care expenditures. JAMA. 2012;307(18):1934-40.

2. Kreek MJ, Bart G, Lilly C, et al. Pharmacogenetics and human molecular genetics of opiate and cocaine addictions and their treatments. Pharmacol Rev. 2005;57(1):1-26.

3. Behnke M, Smith VC. Committee on Substance Abuse. Prenatal substance abuse: short-and long-term effects on the exposed fetus. Pediatrics. 2013;131(3):e1009-24.

4. Seligman NS, Salva N, Hayes EJ, et al. Predicting length of treatment for neonatal abstinence syndrome in methadoneexposed neonates. Am J Obstet Gynecol. 2008;199(4):396. e391-7.
5. Wachman EM, Hayes MJ, Brown MS, et al. Association of OPRM1 and COMT single-nucleotide polymorphisms with hospital length of stay and treatment of neonatal abstinence syndrome. JAMA. 2013;309(17):1821-1827.

6. Wachman EM, Hayes MJ, Lester BM, et al. Epigenetic variation in the mu-opioid receptor gene in infants with neonatal abstinence syndrome. J Pediatr 2014;165(3):472-8.

7. Hudak ML, Tan RC. Neonatal drug withdrawal. Pediatrics. 2012;129(2):e540-60.

8. Mehta A, Forbes KD, Kuppala VS. Neonatal abstinence syndrome management from prenatal counseling to postdischarge follow-up care: Results of a national survey. Hospital Pediatrics. 2013;3(4):317-23.

9. O'Grady MJ, Hopewell J, White MJ. Management of neonatal abstinence syndrome: a national survey and review of practice. Arch Dis Child - Fetal Neonatal Ed. 2009;94(4):249-52.

10. Sarkar S, Donn SM. Management of neonatal abstinence syndrome in neonatal intensive care units: a national survey. J Perinatol. 2006;26(1):15-7.

11. Finnegan LP, Connaughton JFJ, Kron RE, et al. Neonatal abstinence syndrome: assessment and management. Addict Dis. $1975 ; 2: 141-58$

12. Eidelman AI, Schanler RJ, Johnston M, et al. Breastfeeding and the use of human milk. Pediatrics. 2012;129(3):e827-41.

13. Billeaud C, Guillet J, Sandler B. Gastric emptying in infants with or without gastro-oesophageal reflux according to the type of milk. Eur J Clin Nutr. 1990;44(8):577-83.

14. Cavell B. Gastric emptying in infants fed human milk or infant formula. Acta Paediatrica. 1981;70(2):639-41.

15. Shulman RJ, Schanler RJ, Lau C, et al. Early feeding, feeding tolerance, and lactase activity in preterm infants. [J Pediatr. 1998;133(5):645-9.

16. Abdel-Latif ME, Pinner J, Clews S, et al. Effects of breast milk on the severity and outcome of neonatal abstinence syndrome among infants of drug-dependent mothers. Pediatrics. 2006;117(6):e1163-9.

17. Dryden C, Young D, Hepburn M, et al. Maternal methadone use in pregnancy: factors associated with the development of neonatal abstinence syndrome and implications for healthcare resources. J Obstet Gynaecol. 2009;116(5):665-71.

18. McQueen KA, Murphy-Oikonen J, Gerlach K, et al. The impact of infant feeding method on neonatal abstinence scores of methadone-exposed infants. Adv Neonatal Care. 2011;11(4):282-90.

19. TIP WI. Improving treatment for drug-exposed infants Treatment Improvement Protocol (TIP) series 5. 2010.

20. Jansson LM, Choo R, Velez ML, et al. Methadone maintenance and breastfeeding in the neonatal period. Pediatrics. 2008;121(1):106-14. 
21. Swortwood MJ, Scheidweiler KB, Barnes AJ, et al. Simultaneous quantification of buprenorphine, naloxone and phase I and II metabolites in plasma and breastmilk by liquid chromatography-tandem mass spectrometry. Journal of Chromatography A. 2016;1446:70-7.
22. Ilett KF, Hackett LP, Gower S, et al. Estimated dose exposure of the neonata to buprenorphine and its metabolite norbuprenorphine via breastmilk during maternal muprenorphine substitution treatment. Breastfeed Med. 2012;7(4):269-74.

\section{*Correspondence to:}

Paula Radmacher

Department of Pediatrics

University of Louisville School of Medicine

USA

E-mail: paula.radmacher@louisville.edu 